\title{
Speech-Driven Text Retrieval: Using Target IR Collections for Statistical Language Model Adaptation in Speech Recognition
}

\author{
Atsushi Fujii ${ }^{1}$, Katunobu Itou ${ }^{2}$, and Tetsuya Ishikawa ${ }^{1}$ \\ 1 University of Library and Information Science \\ 1-2 Kasuga, Tsukuba, 305-8550, Japan \\ \{fujii,ishikawa\}@ulis.ac.jp \\ 2 National Institute of Advanced Industrial Science and Technology \\ 1-1-1 Chuuou Daini Umezono, Tsukuba, 305-8568, Japan \\ itou@ni.aist.go.jp
}

\begin{abstract}
Speech recognition has of late become a practical technology for real world applications. Aiming at speech-driven text retrieval, which facilitates retrieving information with spoken queries, we propose a method to integrate speech recognition and retrieval methods. Since users speak contents related to a target collection, we adapt statistical language models used for speech recognition based on the target collection, so as to improve both the recognition and retrieval accuracy. Experiments using existing test collections combined with dictated queries showed the effectiveness of our method.
\end{abstract}

\section{Introduction}

Automatic speech recognition, which decodes human voice to generate transcriptions, has of late become a practical technology. It is feasible that speech recognition is used in real world computer-based applications, specifically, those associated with human language. In fact, a number of speech-based methods have been explored in the information retrieval community, which can be classified into the following two fundamental categories:

- spoken document retrieval, in which written queries are used to search speech (e.g., broadcast news audio) archives for relevant speech information $[5,6$, $15-17,19,20]$,

- speech-driven (spoken query) retrieval, in which spoken queries are used to retrieve relevant textual information $[2,3]$.

Initiated partially by the TREC-6 spoken document retrieval (SDR) track [4], various methods have been proposed for spoken document retrieval. However, a relatively small number of methods have been explored for speech-driven text retrieval, although they are associated with numerous keyboard-less retrieval applications, such as telephone-based retrieval, car navigation systems, and userfriendly interfaces. 
Barnett et al. [2] performed comparative experiments related to speech-driven retrieval, where an existing speech recognition system was used as an input interface for the INQUERY text retrieval system. They used as test inputs 35 queries collected from the TREC 101-135 topics, dictated by a single male speaker. Crestani [3] also used the above 35 queries and showed that conventional relevance feedback techniques marginally improved the accuracy for speech-driven text retrieval.

These above cases focused solely on improving text retrieval methods and did not address problems of improving speech recognition accuracy. In fact, an existing speech recognition system was used with no enhancement. In other words, speech recognition and text retrieval modules were fundamentally independent and were simply connected by way of an input/output protocol.

However, since most speech recognition systems are trained based on specific domains, the accuracy of speech recognition across domains is not satisfactory. Thus, as can easily be predicted, in cases of Barnett et al. [2] and Crestani [3], a relatively high speech recognition error rate considerably decreased the retrieval accuracy. Additionally, speech recognition with a high accuracy is crucial for interactive retrieval.

Motivated by these problems, in this paper we integrate (not simply connect) speech recognition and text retrieval to improve both recognition and retrieval accuracy in the context of speech-driven text retrieval.

Unlike general-purpose speech recognition aimed to decode any spontaneous speech, in the case of speech-driven text retrieval, users usually speak contents associated with a target collection, from which documents relevant to their information need are retrieved. In a stochastic speech recognition framework, the accuracy depends primarily on acoustic and language models [1]. While acoustic models are related to phonetic properties, language models, which represent linguistic contents to be spoken, are strongly related to target collections. Thus, it is intuitively feasible that language models have to be produced based on target collections.

To sum up, our belief is that by adapting a language model based on a target IR collection, we can improve the speech recognition and text retrieval accuracy, simultaneously.

Section 2 describes our prototype speech-driven text retrieval system, which is currently implemented for Japanese. Section 3 elaborates on comparative experiments, in which existing test collections for Japanese text retrieval are used to evaluate the effectiveness of our system.

\section{System Description}

\subsection{Overview}

Figure 1 depicts the overall design of our speech-driven text retrieval system, which consists of speech recognition, text retrieval and adaptation modules. We explain the retrieval process based on this figure. 
In the off-line process, the adaptation module uses the entire target collection (from which relevant documents are retrieved) to produce a language model, so that user speech related to the collection can be recognized with a high accuracy. On the other hand, an acoustic model is produced independent of the target collection.

In the on-line process, given an information need spoken by a user, the speech recognition module uses the acoustic and language models to generate a transcription for the user speech. Then, the text retrieval module searches the collection for documents relevant to the transcription, and outputs a specific number of top-ranked documents according to the degree of relevance, in descending order.

These documents are fundamentally final outputs. However, in the case where the target collection consists of multiple domains, a language model produced in the off-line adaptation process is not necessarily precisely adapted to a specific information need. Thus, we optionally use top-ranked documents obtained in the initial retrieval process for an on-line adaptation, because these documents are associated with the user speech more than the entire collection. We then reperform speech recognition and text retrieval processes to obtain final outputs.

In other words, our system is based on the two-stage retrieval principle [8], where top-ranked documents retrieved in the first stage are intermediate results, and are used to improve the accuracy for the second (final) stage. From a different perspective, while the off-line adaptation process produces the global language model for a target collection, the on-line adaptation process produces a local language model based on the user speech.

In the following sections, we explain speech recognition, adaptation, and text retrieval modules in Figure 1, respectively.

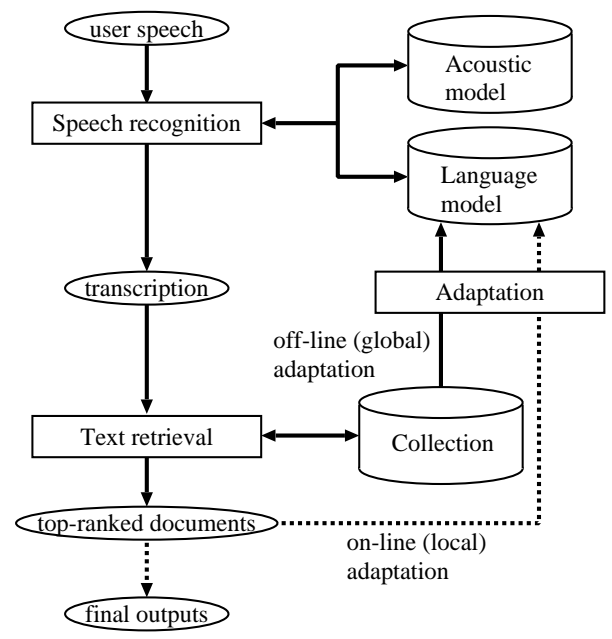

Fig. 1. The overall design of our speech-driven text retrieval system. 


\subsection{Speech Recognition}

The speech recognition module generates word sequence $W$, given phoneme sequence $X$. In the stochastic speech recognition framework, the task is to output the $W$ maximizing $P(W \mid X)$, which is transformed as in equation (1) through use of the Bayesian theorem.

$$
\arg \max _{W} P(W \mid X)=\arg \max _{W} P(X \mid W) \cdot P(W)
$$

Here, $P(X \mid W)$ models a probability that word sequence $W$ is transformed into phoneme sequence $X$, and $P(W)$ models a probability that $W$ is linguistically acceptable. These factors are usually called acoustic and language models, respectively.

For the speech recognition module, we use the Japanese dictation toolkit $[7]^{1}$, which includes the "Julius" recognition engine and acoustic/language models trained based on newspaper articles. This toolkit also includes development softwares, so that acoustic and language models can be produced and replaced depending on the application. While we use the acoustic model provided in the toolkit, we use new language models produced by way of the adaptation process (see Section 2.3).

\subsection{Language Model Adaptation}

The basis of the adaptation module is to produce a word-based $N$-gram (in our case, a combination of bigram and trigram) model by way of source documents.

In the off-line (global) adaptation process, we use the ChaSen morphological analyzer [10] to extract words from the entire target collection, and produce the global $N$-gram model.

On the other hand, in the on-line (local) adaptation process, only top-ranked documents retrieved in the first stage are used as source documents, from which word-based $N$-grams are extracted as performed in the off-line process. However, unlike the case of the off-line process, we do not produce the entire language model. Instead, we re-estimate only statistics associated with top-ranked documents, for which we use the MAP (Maximum A-posteriori Probability) estimation method [9].

Although the on-line adaptation theoretically improves the retrieval accuracy, for real-time usage, the trade-off between the retrieval accuracy and computational time required for the on-line process has to be considered.

Our method is similar to the one proposed by Seymore and Rosenfeld [14] in the sense that both methods adapt language models based on a small number of documents related to a specific domain (or topic). However, unlike their method, our method does not require corpora manually annotated with topic tags.

\footnotetext{
${ }^{1}$ http://winnie.kuis.kyoto-u.ac.jp/dictation/
} 


\subsection{Text Retrieval}

The text retrieval module is based on an existing probabilistic retrieval method [13], which computes the relevance score between the transcribed query and each document in the collection. The relevance score for document $i$ is computed based on equation (2).

$$
\sum_{t}\left(\frac{T F_{t, i}}{\frac{D L_{i}}{\text { avglen }}+T F_{t, i}} \cdot \log \frac{N}{D F_{t}}\right)
$$

Here, $t$ 's denote terms in transcribed queries. $T F_{t, i}$ denotes the frequency that term $t$ appears in document $i$. $D F_{t}$ and $N$ denote the number of documents containing term $t$ and the total number of documents in the collection. $D L_{i}$ denotes the length of document $i$ (i.e., the number of characters contained in $i$ ), and avglen denotes the average length of documents in the collection.

We use content words extracted from documents as terms, and perform a word-based indexing. For this purpose, we use the ChaSen morphological analyzer [10] to extract content words. We extract terms from transcribed queries using the same method.

\section{Experimentation}

\subsection{Test Collections}

We investigated the performance of our system based on the NTCIR workshop evaluation methodology, which resembles the one in the TREC ad hoc retrieval track. In other words, each system outputs 1,000 top documents, and the TREC evaluation software was used to plot recall-precision curves and calculate noninterpolated average precision values.

The NTCIR workshop was held twice (in 1999 and 2001), for which two different test collections were produced: the NTCIR-1 and 2 collections $[11,12]^{2}$. However, since these collections do not include spoken queries, we asked four speakers (two males/females) to dictate information needs in the NTCIR collections, and simulated speech-driven text retrieval.

The NTCIR collections include documents collected from technical papers published by 65 Japanese associations for various fields. Each document consists of the document ID, title, name(s) of author(s), name/date of conference, hosting organization, abstract and author keywords, from which we used titles, abstracts and keywords for the indexing. The number of documents in the NTCIR-1 and 2 collections are 332,918 and 736,166, respectively (the NTCIR-1 documents are a subset of the NTCIR-2).

The NTCIR-1 and 2 collections also include 53 and 49 topics, respectively. Each topic consists of the topic ID, title of the topic, description, narrative. Figure 2 shows an English translation for a fragment of the NTCIR topics ${ }^{3}$,

${ }^{2}$ http://research.nii.ac.jp/ ${ }^{\sim}$ ntcadm/index-en.html

3 The NTCIR-2 collection contains Japanese topics and their English translations. 
where each field is tagged in an SGML form. In general, titles are not informative for the retrieval. On the other hand, narratives, which usually consist of several sentences, are too long to speak. Thus, only descriptions, which consist of a single phrase and sentence, were dictated by each speaker, so as to produce four different sets of 102 spoken queries.

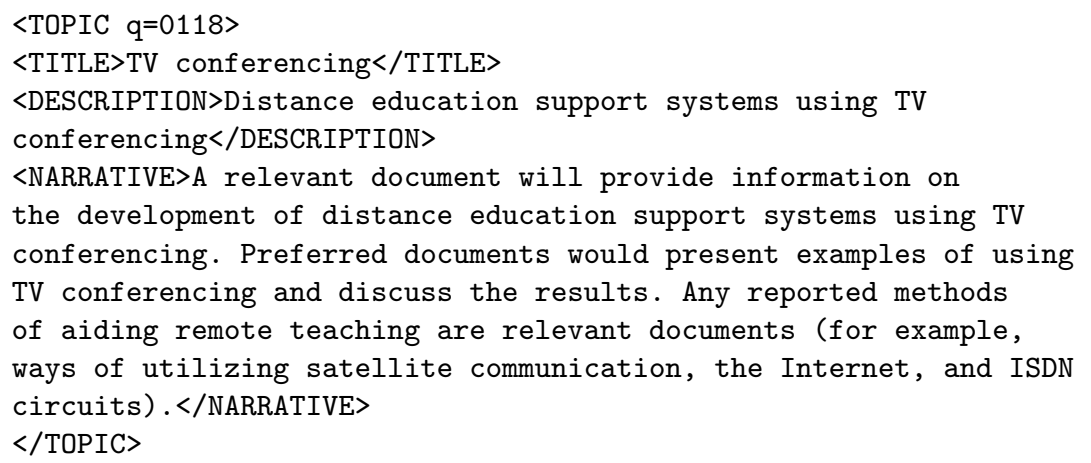

Fig. 2. An English translation for an example topic in the NTCIR collections.

In the NTCIR collections, relevance assessment was performed based on the pooling method [18]. First, candidates for relevant documents were obtained with multiple retrieval systems. Then, for each candidate document, human expert(s) assigned one of three ranks of relevance: "relevant," "partially relevant" and "irrelevant." The NTCIR-2 collection also includes "highly relevant" documents. In our evaluation, "highly relevant" and "relevant" documents were regarded as relevant ones.

\subsection{Comparative Evaluation}

In order to investigate the effectiveness of the off-line language model adaptation, we compared the performance of the following different retrieval methods:

- text-to-text retrieval, which used written descriptions as queries, and can be seen as the perfect speech-driven text retrieval,

- speech-driven text retrieval, in which a language model produced based on the NTCIR-2 collection was used,

- speech-driven text retrieval, in which a language model produced based on ten years worth of Mainichi Shimbun Japanese newspaper articles (19912000) was used.

The only difference in producing two different language models (i.e., those based on the NTCIR-2 collection and newspaper articles) are the source documents. 
In other words, both language models have the same vocabulary size $(20,000)$, and were produced using the same softwares.

Table 1 shows statistics related to word tokens/types in two different source corpora for language modeling, where the line "Coverage" denotes the ratio of word tokens contained in the resultant language model. Most of word tokens were covered in both language models.

Table 1. Statistics associated with source words for language modeling.

\begin{tabular}{lcc}
\hline \hline & NTCIR & News \\
\hline \# of Types & $454 \mathrm{~K}$ & $315 \mathrm{~K}$ \\
\# of Tokens & $175 \mathrm{M}$ & $262 \mathrm{M}$ \\
Coverage & $97.9 \%$ & $96.5 \%$ \\
\hline
\end{tabular}

In cases of speech-driven text retrieval methods, queries dictated by four speakers were used individually. Thus, in practice we compared nine different retrieval methods. Although the Julius decoder outputs more than one transcription candidate for a single speech input, we used only the one with the greatest probability score. The results did not significantly change depending on whether or not we used lower-ranked transcriptions as queries.

Table 2 shows the non-interpolated average precision values and word error rate in speech recognition, for different retrieval methods. As with existing experiments for speech recognition, word error rate (WER) is the ratio between the number of word errors (i.e., deletion, insertion, and substitution) and the total number of words. In addition, we also investigated error rate with respect to query terms (i.e., keywords used for retrieval), which we shall call "term error rate (TER)."

In Table 2, the first line denotes results of the text-to-text retrieval, which were relatively high compared with existing results reported in the NTCIR workshops $[11,12]$.

The remaining lines denote results of speech-driven text retrieval combined with the NTCIR-based language model (lines 2-5) and the newspaper-based model (lines 6-9), respectively. Here, "Mx" and "Fx" denote male/female speakers, respectively. Suggestions which can be derived from these results are as follows.

First, for both language models, results did not significantly change depending on the speaker. The best average precision values for speech-driven text retrieval were obtained with a combination of queries dictated by a male speaker (M1) and the NTCIR-based language model, which were approximately $80 \%$ of those with the text-to-text retrieval.

Second, by comparing results of different language models for each speaker, one can see that the NTCIR-based model significantly decreased WER and TER obtained with the newspaper-based model, and that the retrieval method using 
Table 2. Results for different retrieval methods (AP: average precision, WER: word error rate, TER: term error rate).

\begin{tabular}{lcccccc}
\hline \hline \multirow{2}{*}{ Method } & \multicolumn{3}{c}{ NTCIR-1 } & \multicolumn{3}{c}{ NTCIR-2 } \\
\cline { 2 - 7 } & AP & WER & TER & AP & WER & TER \\
\hline Text & 0.3320 & - & - & 0.3118 & - & - \\
M1 (NTCIR) & 0.2708 & 0.1659 & 0.2190 & 0.2504 & 0.1532 & 0.2313 \\
M2 (NTCIR) & 0.2471 & 0.2034 & 0.2381 & 0.2114 & 0.2180 & 0.2799 \\
F1 (NTCIR) & 0.2276 & 0.1961 & 0.2857 & 0.1873 & 0.1885 & 0.2500 \\
F2 (NTCIR) & 0.2642 & 0.1477 & 0.2222 & 0.2376 & 0.1635 & 0.2388 \\
M1 (News) & 0.1076 & 0.3547 & 0.5143 & 0.0790 & 0.3594 & 0.5149 \\
M2 (News) & 0.1257 & 0.4044 & 0.5460 & 0.0691 & 0.5022 & 0.6343 \\
F1 (News) & 0.1156 & 0.3801 & 0.5238 & 0.0798 & 0.4418 & 0.5709 \\
F2 (News) & 0.1225 & 0.3317 & 0.5016 & 0.0917 & 0.4080 & 0.5858 \\
\hline
\end{tabular}

the NTCIR-based model significantly outperformed one using the newspaperbased model. In addition, these results were observable, irrespective of the speaker. Thus, we conclude that adapting language models based on target collections was quite effective for speech-driven text retrieval.

Third, TER was generally higher than WER irrespective of the speaker. In other words, speech recognition for content words was more difficult than functional words, which were not contained in query terms.

We analyzed transcriptions for dictated queries, and found that speech recognition error was mainly caused by the out-of-vocabulary problem. In the case where major query terms are mistakenly recognized, the retrieval accuracy substantially decreases. In addition, descriptions in the NTCIR topics often contain expressions which do not appear in the documents, such as "I want papers about..." Although these expressions usually do not affect the retrieval accuracy, misrecognized words affect the recognition accuracy for remaining words including major query terms. Consequently, the retrieval accuracy decreases due to the partial misrecognition.

Finally, we investigated the trade-off between recall and precision. Figures 3 and 4 show recall-precision curves of different retrieval methods, for the NTCIR1 and 2 collections, respectively. In these figures, the relative superiority for precision values due to different language models in Table 2 was also observable, regardless of the recall.

However, the effectiveness of the on-line adaptation remains an open question and needs to be explored.

\section{Conclusion}

Aiming at speech-driven text retrieval with a high accuracy, we proposed a method to integrate speech recognition and text retrieval methods, in which target text collections are used to adapt statistical language models for speech 


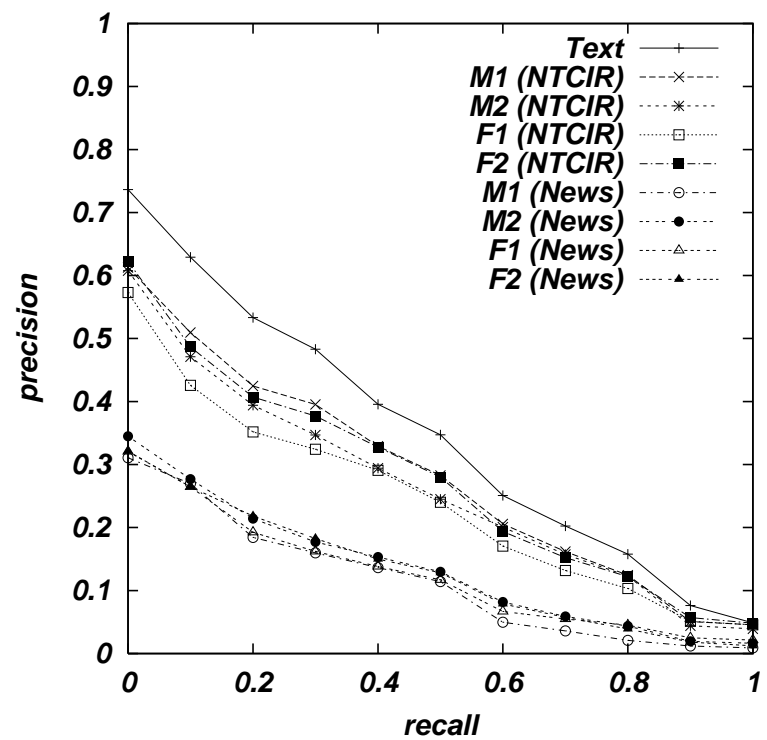

Fig. 3. Recall-precision curves for different retrieval methods using the NTCIR-1 collection.

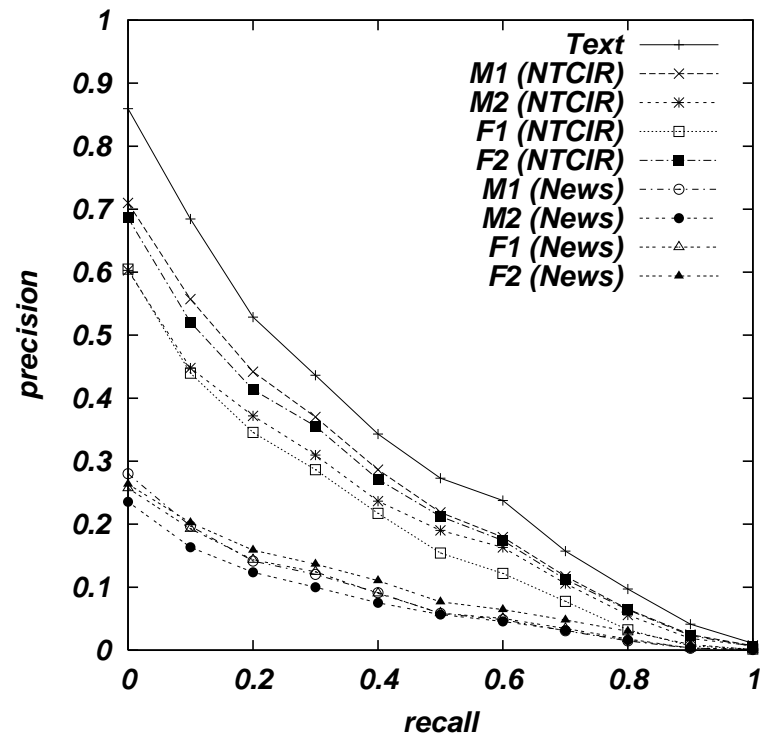

Fig. 4. Recall-precision curves for different retrieval methods using the NTCIR-2 collection. 
recognition. We also showed the effectiveness of our method by way of experiments, where dictated information needs in the NTCIR collections were used as queries to retrieve technical abstracts. Future work would include experiments on various collections, such as newspaper articles and Web pages.

\section{Acknowledgments}

The authors would like to thank the National Institute of Informatics for their support with the NTCIR collections.

\section{References}

1. L. R. Bahl, F. Jelinek, and R. L. Mercer. A maximum likelihood approach to continuous speech recognition. IEEE Transactions on Pattern Analysis and Machine Intelligence, 5(2):179-190, 1983.

2. J. Barnett, S. Anderson, J. Broglio, M. Singh, R. Hudson, and S. W. Kuo. Experiments in spoken queries for document retrieval. In Proceedings of Eurospeech97, pages $1323-1326,1997$.

3. F. Crestani. Word recognition errors and relevance feedback in spoken query processing. In Proceedings of the Fourth International Conference on Flexible Query Answering Systems, pages 267-281, 2000.

4. J. S. Garofolo, E. M. Voorhees, V. M. Stanford, and K. S. Jones. TREC-6 1997 spoken document retrieval track overview and results. In Proceedings of the 6th Text REtrieval Conference, pages 83-91, 1997.

5. S. Johnson, P. Jourlin, G. Moore, K. S. Jones, and P. Woodland. The Cambridge University spoken document retrieval system. In Proceedings of ICASSP'99, pages 49-52, 1999.

6. G. Jones, J. Foote, K. S. Jones, and S. Young. Retrieving spoken documents by combining multiple index sources. In Proceedings of the 19th Annual International ACM SIGIR Conference on Research and Development in Information Retrieval, pages 30-38, 1996.

7. T. Kawahara, A. Lee, T. Kobayashi, K. Takeda, N. Minematsu, S. Sagayama, K. Itou, A. Ito, M. Yamamoto, A. Yamada, T. Utsuro, and K. Shikano. Free software toolkit for Japanese large vocabulary continuous speech recognition. In Proceedings of the 6th International Conference on Spoken Language Processing, pages 476-479, 2000.

8. K. Kwok and M. Chan. Improving two-stage ad-hoc retrieval for short queries. In Proceedings of the 21st Annual International ACM SIGIR Conference on Research and Development in Information Retrieval, pages 250-256, 1998.

9. H. Masataki, Y. Sagisaka, K. Hisaki, and T. Kawahara. Task adaptation using MAP estimation in n-gram language modeling. In Proceedings of ICASSP'97, pages $783-786,1997$.

10. Y. Matsumoto, A. Kitauchi, T. Yamashita, Y. Hirano, H. Matsuda, and M. Asahara. Japanese morphological analysis system ChaSen version 2.0 manual 2nd edition. Technical Report NAIST-IS-TR99009, NAIST, 1999.

11. National Center for Science Information Systems. Proceedings of the 1st NTCIR Workshop on Research in Japanese Text Retrieval and Term Recognition, 1999. 
12. National Institute of Informatics. Proceedings of the 2nd NTCIR Workshop Meeting on Evaluation of Chinese 83 Japanese Text Retrieval and Text Summarization, 2001.

13. S. Robertson and S. Walker. Some simple effective approximations to the 2-poisson model for probabilistic weighted retrieval. In Proceedings of the 17th Annual International ACM SIGIR Conference on Research and Development in Information Retrieval, pages 232-241, 1994.

14. K. Seymore and R. Rosenfeld. Using story topics for language model adaptation. In Proceedings of Eurospeech97, 1997.

15. P. Sheridan, M. Wechsler, and P. Schäuble. Cross-language speech retrieval: Establishing a baseline performance. In Proceedings of the 20th Annual International ACM SIGIR Conference on Research and Development in Information Retrieval, pages 99-108, 1997.

16. A. Singhal and F. Pereira. Document expansion for speech retrieval. In Proceedings of the 22nd Annual International ACM SIGIR Conference on Research and Development in Information Retrieval, pages 34-41, 1999.

17. S. Srinivasan and D. Petkovic. Phonetic confusion matrix based spoken document retrieval. In Proceedings of the 23rd Annual International ACM SIGIR Conference on Research and Development in Information Retrieval, pages 81-87, 2000.

18. E. M. Voorhees. Variations in relevance judgments and the measurement of retrieval effectiveness. In Proceedings of the 21st Annual International ACM SIGIR Conference on Research and Development in Information Retrieval, pages 315-323, 1998.

19. M. Wechsler, E. Munteanu, and P. Schäuble. New techniques for open-vocabulary spoken document retrieval. In Proceedings of the 21st Annual International ACM SIGIR Conference on Research and Development in Information Retrieval, pages 20-27, 1998.

20. S. Whittaker, J. Hirschberg, J. Choi, D. Hindle, F. Pereira, and A. Singhal. SCAN: Designing and evaluating user interfaces to support retrieval from speech archives. In Proceedings of the 22nd Annual International ACM SIGIR Conference on Research and Development in Information Retrieval, pages 26-33, 1999. 